\title{
Efficient Time-Domain DBP using Random Step-Size and Multi-Band Quantization
}

\author{
C. S. Martins ${ }^{1}$, L. Bertignono ${ }^{2}$, A. Nespola ${ }^{3}$, A. Carena ${ }^{2}$, F. P. Guiomar ${ }^{2}$ and A. N. Pinto ${ }^{1}$ \\ ${ }^{I}$ Department of Electronics, Telecommunications and Informatics, University of Aveiro and Instituto de \\ Telecomunicações, 3810-193, Aveiro,Portugal, csmartins@av.it.pt \\ ${ }^{2}$ DET, Politecnico di Torino, Corso Duca degli Abruzzi, 24, 10129, Torino, Italy, fernando.guiomar@ polito.it \\ ${ }^{3}$ Istituto Superiore Mario Boella, via Pier Carlo Boggio 61, 10138 Torino, Italy, nespola@ismb.it
}

\begin{abstract}
Employing step-size randomization and multi-band quantization, we propose a reduced complexity time-domain (TD) digital backpropagation (DBP) and experimentally demonstrate penalty-free operation at an average number of $\sim 4$ bits per FIR coefficient. OCIS codes: (060.2360) Fiber optics links and subsystems; (060.1660) Coherent communications.
\end{abstract}

\section{Introduction}

Since the pioneer works on fiber nonlinearity mitigation via digital backpropagation (DBP) [1], the research in this field has seen notable developments in terms of complexity reduction employing a wide variety of algorithms [2]. However, high computational effort and power consumption still represent a strong practical barrier towards an efficient hardware implementation and commercial deployment of DBP.

In the last few years, several DBP-based techniques with reduced complexity have been proposed $[3,4]$, in which the linear operator is preferably implemented in frequency-domain, favoring from efficient FFT-based processing. However, the iterative application of FFT/IFFT pairs for each DBP step remains a major complexity bottleneck. Alternatively, full time-domain (TD) DBP FIR-based approaches have also been demonstrated [5-7], in which case the computational optimization burden is shifted towards the design of efficient FIR filters. Recently, advanced strategies for alleviating the bit precision requirements of TD-DBP have been proposed [5,6], exploring aggressive coefficient quantization approaches. These efforts have demonstrated through simulations that a penalty-free TD-DBP implementation is achievable using 9-bit fixed-point precision [6]. However, since the practical feasibility of TD-DBP highly depends on a low bit precision implementation, it is imperative to further decrease this requirement.

In this paper, we propose a novel TD-DBP approach based on multi-band quantization and step-size randomization techniques, enabling penalty-free operation at an average $\sim 4$ bits of precision for the FIR coefficients. These results are experimentally demonstrated by long-haul transmission of 21-channel PM-16QAM, with intra-channel TD-DBP yielding $\sim 0.5 \mathrm{~dB}$ of Q-factor gain $\left(Q_{\text {gain }}\right)$ over chromatic dispersion equalization (CDE).

\section{Relaxing the Hardware Requirements of Time-Domain DBP}

One of the key challenges towards the minimization of bit precision requirements in TD-DBP is the fact that DBP techniques typically involve a large number of concatenated steps, which greatly enhances error propagation due to coarse quantization of the FIR coefficients. This error propagation between steps becomes even more limiting if the same set of coefficients and the same quantization process are applied among all steps, in which case the quantization error propagates coherently, strongly damaging the signal. In order to partially circumvent this issue, advanced strategies for FIR quantization have proposed in [5,6], either resorting to dithering of the FIR coefficients [5] or joint optimization of FIR filter pairs [6]. In this work, we propose a simpler approach based on the randomization of the DBP step-size (R-TD-DBP) around a small interval, thus allowing to effectively decorrelate the quantization error among steps, without the need to introduce dithering noise or performing complex joint FIR optimization. The basic idea behind this technique is to impose a random step-size, $h$, in TD-DBP over each iteration, such that a slightly different amount of dispersion is compensated at each step. This in turn, imposes that a different set of FIR coefficients are required for the linear equalization in each iteration, enabling to partially decorrelate the generated quantization noises on each step. Fig. 1a illustrates the concept of R-TD-DBP using one step per span, where the step-size is randomized over a given interval, $R$. The $\hat{D}$ and $\hat{N}$ operators in Fig. 1a correspond to the well known linear (chromatic dispersion and attenuation) and nonlinear DBP steps, respectively. Note that this randomization interval should be large enough to enable effective decorrelation of the FIR filters, while in the other hand it has to remain small enough to not degrade the DBP performance by displacing the optimum position of the nonlinear operator, $\hat{N}_{\text {opt }}$. In this work we have found that a randomization interval of $10 \%$ of the span length $(\sim 10.8 \mathrm{~km})$ provided an excellent compromise between these two requirements for the considered system under test. 

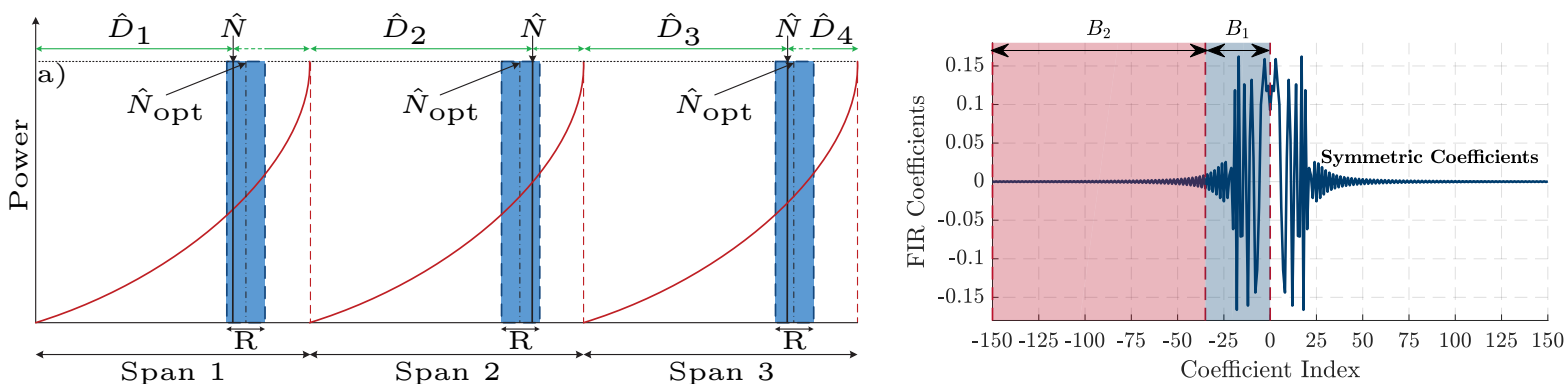

Fig. 1: a) Random step-size TD-DBP implementation scheme; b) Multi-band quantization applied to the real part of FIR coefficients. The single band FIR coefficients are split into two bands, $B_{1}$ and $B_{2}$.

As shown in Fig. 1b, the distribution of coefficients of the FIR filter can be divided into two rather distinct bands: i) the central part of the FIR (band $B_{1}$ ), whose coefficients vary over a wide range of values, and ii) the FIR tail (band $B_{2}$ ), where the coefficients vary within a much restricted range. It therefore becomes apparent that an efficient quantization of the FIR filter coefficients would benefit from a separate treatment of these two bands. Based on this observation, we propose a dual-band quantization process, in which bands $B_{1}$ and $B_{2}$ are separately renormalized and quantized as,

$$
c_{\mathrm{r} / \mathrm{i}}^{Q, j}(k)=\frac{\max \left(\left|c_{\mathrm{r} / \mathrm{i}}^{j}\right|\right)}{\Delta}\left\lfloor\Delta \frac{c_{\mathrm{r} / \mathrm{i}}^{j}}{\max \left(\left|c_{\mathrm{r} / \mathrm{i}}^{j}(k)\right|\right)}\right\rceil, k=0, \ldots, M_{j}-1,
$$

where, $\Delta$ is the quantization factor, $c_{\mathrm{r} / \mathrm{i}}^{Q, j}$ are the real or imaginary parts of quantized FIR coefficients $\left(c^{Q}\right)$ of band $j\left(B_{j}\right)$, respectively, $M_{j}$ is the number of taps in band $B_{j}$ and $\lfloor\cdot 7$ is the nearest integer operation. Since the amplitude range of each band may differ, we can now adjust the $\Delta$ value to a given band according to its respective amplitude range. Considering the dual-band approach depicted in Fig. 1b, we can then expect that by renormalizing and independently quantizing the tail band $\left(B_{2}\right)$, we should be able to significantly reduce the bit precision requirements there. Note that this renormalization process implies an additional real multiplication in the FIR structure. Nevertheless, since this multiplication is common to all coefficients inside the band, it can be applied after summing up all their contributions, and thus its added complexity is negligible. Also note that, to take advantage of the FIR filter symmetry, we consider as well a folded delay line FIR structure, enabling to reduce the number of effective coefficients by half [8].

\section{Experimental Results}

In this section, we provide the experimental validation of the proposed techniques considering the laboratorial setup depicted in Fig. 2. At the transmitter-side, a $64 \mathrm{Gsa} / \mathrm{s}$ digital to analog converter (DAC) generates the analog I and Q components of the channel under test (CUT) for both polarizations, modulated by an external cavity laser (ECL). A 4-port DAC is utilized to generate the analog I and Q components of 20 interferer channels, modulated by distributed feedback (DFB) lasers arranged in 10 odd and even carriers. A polarization multiplexing emulator (PME) is utilized to perform optical polarization multiplexing for the interferer channels. The transmission link comprises a recirculatingloop controlled by acousto-optical modulators (AOMs) and is composed by four spans of pure silica core fiber (PSCF) with length of $108 \mathrm{~km}$, characterized by $0.16 \mathrm{~dB} / \mathrm{km}$ of attenuation and $20.17 \mathrm{ps} /(\mathrm{nm} \cdot \mathrm{km})$ of dispersion. EDFAs with $5.4 \mathrm{~dB}$ of noise figure are utilized to recover the loss, and a gain-equalizer (GEQ) followed by the polarization scrambler (PolScr) are utilized to flatten the optical gain and average the polarization effects. At the receiver side, the CUT is filtered by a tunable optical filter (TOF), and then coherently detected. The electrical signal is sampled by a $50 \mathrm{Gsa} / \mathrm{s}$ oscilloscope with $33 \mathrm{GHz}$ electrical bandwidth and followed by offline post-processing. The DSP includes linear (CDE) or nonlinear (TD-DBP) compensation, adaptive equalization, frequency mismatch, phase estimation and finally Q-factor estimation directly obtained from bit error rate (BER) counting.

We initiate our analysis by optimizing the TD-DBP performance in terms of launched power, number of steps

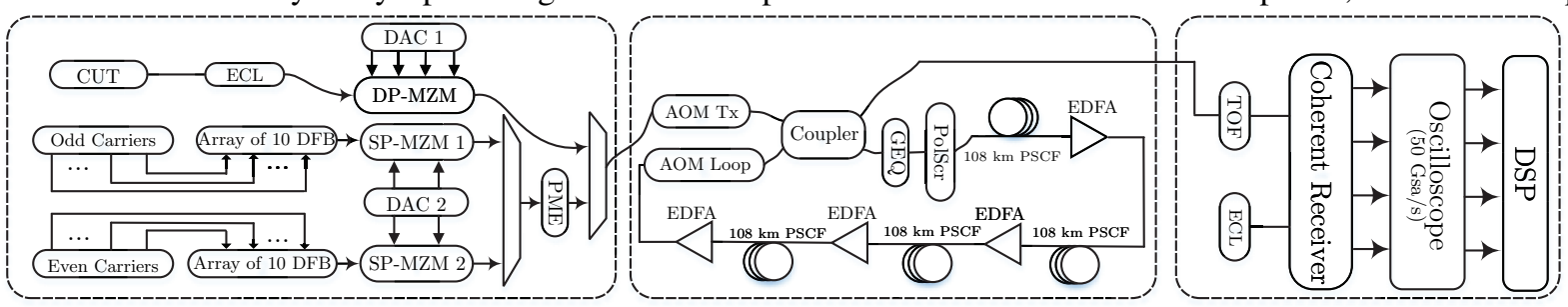

Fig. 2: Experimental setup of $21 \times 32$ GBd WDM PM-16QAM transmission system. 

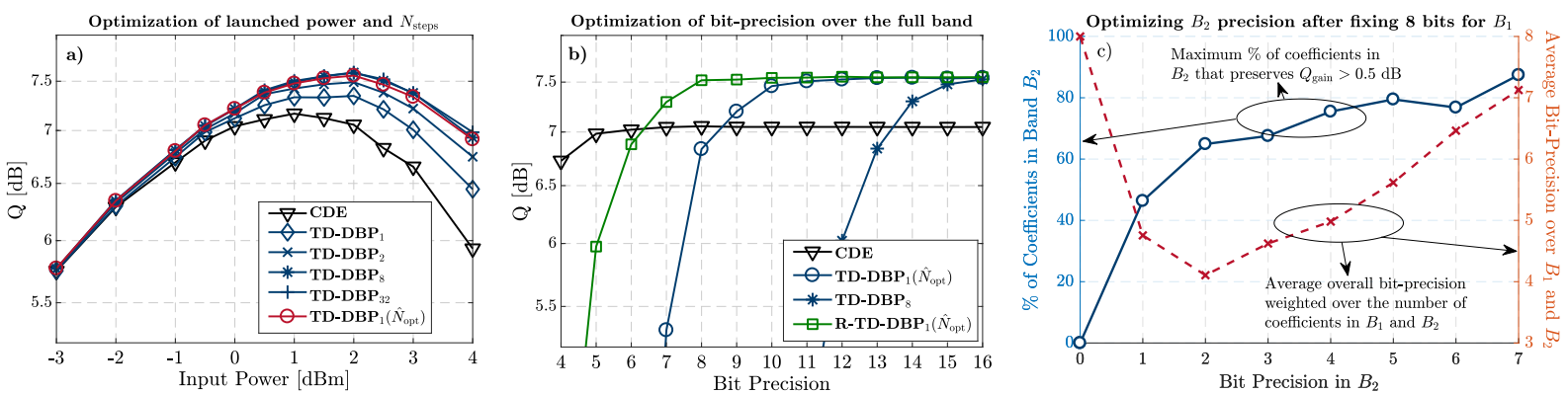

Fig. 3: a) BER versus input power considering different number of steps per span; b) BER versus bit precision for different step-size configurations; c) Optimization of multi-band quantization to minimize the weighted average bit precision requirements.

per span, $N_{\text {steps }}$, and position of the nonlinear operator. As for nomenclature, we define the following variants of TD-DBP: TD-DBP $N_{N_{\text {steps }}}$ as the standard TD-DBP applied with $N_{\text {steps }}$ per span, ii) TD-DBP steps $\left._{N_{\text {spt }}}\right)$ as the TD$\mathrm{DBP}_{N_{\text {steps }}}$ algorithm with optimized positioning of the nonlinear operator, and iii) R-TD-DBP ${ }_{N_{\text {steps }}}\left(\hat{N}_{\text {opt }}\right)$ as the TD$\mathrm{DBP}_{N_{\text {steps }}}\left(\hat{N}_{\text {opt }}\right)$ algorithm with randomized step-size for more robust FIR quantization. The experimental results shown in Fig. 3a reveal that, by simply optimizing the position of the nonlinear operator (in this work we found an optimum position for the $\hat{N}$ operator at $\sim 80 \%$ of the span length), we can operate at $1 \mathrm{step} / \mathrm{span}$ with roughly the same accuracy as an 8 -steps/span TD-DBP. This is a major first step towards the reduction of DBP complexity.

Then, we proceed with the optimization of TD-DBP $1\left(\hat{N}_{\text {opt }}\right)$ in terms of bit precision requirements for the FIR filters in the linear step, whose results are shown in Fig. 3b. As expected, increasing the step-size also allows to relax the bit precision requirements, since the quantization errors are propagated over a lower number of steps. Indeed, a reduction of 4 bits is achieved by simply considering TD-DBP $1\left(\hat{N}_{\text {opt }}\right)$ (requiring 11 bits) instead of the standard TD-DBP 8 (requiring 15 bits). After optimizing the number of FIR taps for TD-DBP $1\left(\hat{N}_{\text {opt }}\right)$, we found that a minimum of 151 taps were required to avoid any implementation penalties. To further increase the robustness against FIR coefficient quantization, we proceed with the random step-size distribution, R-TD-DBP $\left(\hat{N}_{\text {opt }}\right)$, which is shown in Fig. $3 \mathrm{~b}$ to effectively reduce the required bit precision down to only 8 bits, without affecting the maximum performance of DBP, which was found to provide $\sim 0.5 \mathrm{~dB}$ of Q-factor gain $\left(Q_{\text {gain }}\right)$ over CDE. Finally, we consider the multi-band quantization approach, MB-R-TD-DBP ${ }_{1}\left(\hat{N}_{\text {opt }}\right)$, with the aim to find out if a reduction of complexity can be achieved by reducing the bit precision in the FIR tail coefficients. In order to do so, we fix the bit precision of band $B_{1}$ (central part of the FIR) to 8 bits, corresponding to the minimum precision requirements obtained from Fig. 3b. Then, we vary the number of quantization bits $(<8)$ allocated to band $B_{2}$ and we find the maximum number coefficients that can be allocated to this band $\left(M_{2}\right)$ while maintaining a $Q_{\text {gain }}$ of $\geq 0.5 \mathrm{~dB}$. These results are shown in Fig. 3c. As expected, when we try to lower number of bits in band $B_{2}$, also the division point between bands in Fig. $1 \mathrm{~b}$ tends to shift towards the tail of the FIR filter, i.e. the ratio between $M_{2} / M$ is reduced. Nevertheless, even for an extreme case of using only 2 bits in band $B_{2}$, still around $65 \%$ of FIR coefficients can be allocated to this band, while the remaining $35 \%$ of coefficients are allocated to band $B_{1}$. This corresponds to an average bit precision of $\sim 4.1$ bits for the full band FIR filter. Note that for 0 bits in $B_{2}$ no coefficients could be allocated to that band, confirming that the impulse response of the FIR could not be further truncated below the considered 151 taps.

\section{Conclusions}

We have proposed a low complexity TD-DBP approach based on step-size randomization and multi-band quantization to enhance the robustness towards FIR coefficient quantization. The proposed technique has been experimentally demonstrated in a $21 \times 32$ GBd WDM PM-16QAM transmission system, enabling to operate at an average number of $\sim 4$ bits per FIR coefficient, without degrading the DBP performance.

This work was supported in part by Fundação para a Ciência e a Tecnologia (FCT) through national funds, and when applicable co-funded by FEDER-PT2020 partnership agreement, under the project UID/EEA/50008/2013 (action SoftTransceiver), by the Ph.D. grant PD/BD/113817/2015 and by the European Commission through a Marie Skłodowska-Curie individual fellowship, project Flex-ON (653412).

\section{References}

[1] E. Ip et al., "Compensation of Dispersion and Nonlinear Impairments Using ..., JLT, vol. 26, no. 20, pp. 3416-3425, 2008.

[2] J. Cartledge et al., "Digital signal processing for fiber nonlinearities," Opt. Express, vol. 25, pp. 1916-1936, 2017.

[3] D. Rafique et al., "Compensation of intra-channel nonlinear fibre impairments using...," Opt. Express vol. 19, no. 10, pp. 9453-9460, 2011.

[4] J. Gonçalves et al., "Nonlinear compensation with DBP aided by ...," Opt. Express vol. 24, pp. 30309-30316, 2016.

[5] C. Fougstedt, et al., "Time-Domain Digital Back Propagation: Algorithm and Finite-Precision ...”, OFC, paper W1G.4, 2017.

[6] C. Fougstedt, et al., "Finite-Precision Optimization of Time-Domain Digital Back Propagation ...", ECOC, paper W.1.D.2, 2017.

[7] L. Zhu, et al., "Complementary FIR Filter Pair for Distributed Impairment Compensation...", PTL, vol. 21, no. 5, pp. 292-294, 2009.

[8] C. S. Martins, et al., "Distributive FIR-Based Chromatic Dispersion Equalization for Coherent...", JLT, vol. 21, no. 34, pp. 5023-5032, 2016. 